\title{
PEG equilibrium partitioning in $\alpha$-hemolysin channel: neutral polymer interaction with channel charges
}

\author{
Marcel Aguilella-Arzo and Vicente M. Aguilella* \\ Laboratory of Molecular Biophysics, Department of Physics, Universitat Jaume I, Castellón, \\ Spain. \\ * corresponding author. aguilell@uji.es
}

\begin{abstract}
We study the interaction of neutral polyethylene glycol (PEG) molecules of different molecular weight (MW) with the charged residues of the $\alpha$-hemolysin channel secreted by $S$. aureus. Previously reported experiments of PEG equilibrium partitioning into this nanopore show that the charge state of the channel changes the ability of PEG entry in a MW-dependent manner. We explain such effect by parameter-free calculations of the PEG self-energy from the channel 3D atomic structure that include repulsive dielectrophoretic and hydrostatic forces on the polymer. We found that the $\mathrm{pH}$-induced shift in the measured free energy of partitioning $\Delta \Delta G_{\text {exp }}$ from single-channel conductance measurements agrees with calculated energy changes $\Delta \Delta E_{\text {calc. Our }}$ results show that PEG-sizing technique may need corrections in the case of charged biological pores.
\end{abstract}

Keywords: PEG; dielectrophoresis; $\alpha$-hemolysin; channel sizing; polymer confinement 


\section{Introduction}

Neutral polymers like polyethylene glycols (PEG) or dextran are often used in the biophysical characterization of protein ion channels. Particularly, in pore sizing, ${ }^{1-17}$ access resistance measurements, ${ }^{1,8,18}$ or to induce osmotic stress in polymer-excluded regions. ${ }^{19-21}$ The use of polymer partitioning into biological nanopores to size the aqueous pore of protein ion channels has become a standard technique and it proved useful as a complement to structural approaches like X-ray diffraction or Cryo-EM. Some of these applications involve the polymer confinement in aqueous pores of protein channels with charged residues. The basic principles of polymer behavior in restricted volumes were established by the pioneers in this field ${ }^{22,23}$ but a good understanding of the physics behind the interaction of polymer molecules with membranes, channel proteins, and other polymer molecules needs further contributions. So far, phenomenological approaches have been mostly used to quantitatively describe polymer equilibrium partitioning in biological pores and scaling theories seem insufficient for an accurate description. Here we aim to contribute to a particular aspect of this problem: the interaction of neutral polymers with charged channel proteins in electrolyte solutions ${ }^{1}$.

A well-known phenomenon relevant to this problem is the polarization of a neutral particle whose dielectric properties differ from those of the surrounding medium in the presence of an electric field. Under inhomogeneous electric fields acting on the particle, this polarization causes a dielectrophoretic force, which is the basis of several biotechnology and biomedical applications involving particle trapping, cell separation, etc. ${ }^{24,25}$. This effect is conceptually equivalent to the energetic penalty of an ion crossing a narrow pore opened in a low dielectric medium (e.g. a lipid bilayer and/or a transmembrane protein) where a charged particle is repelled from a neutral low dielectric surrounding medium. ${ }^{26}$ In addition, whenever a neutral particle perturbs the equilibrium electric double layer near a charged surface, the pressure gradient acts as a net repulsive force that pushes the particle away from that surface. Both the dielectrophoretic force $\left(F_{D}\right)$ and the hydrostatic force $\left(F_{H}\right)$ contribute to exclude a neutral particle from a charged surface provided the polarizability is lower in the particle than in the solution. ${ }^{27}$

Here we focus on the effect of these two forces acting on neutral polymers that partition into the aqueous pore of the $\alpha$-hemolysin (aHL) channel, a toxin secreted by Staphylococcus aureus. The aHL channel has been used for biosensing for more than two decades ${ }^{28,29}$ and it was the starting point for nanopore-based single-molecule mass spectrometry. We take the advantage that its 3D atomic structure and charge distribution are known. ${ }^{30}$ Its mild anionic selectivity at neutral $\mathrm{pH}^{31}$ can be modulated or even reversed by changing the solution acidity. In order to compare our model calculations with the existing experimental data on polymer partitioning we analyze the interaction between the aHL channel and different molecular weight (MW) PEGs. The goal is showing that, in the absence of specific effects like polymer-cation binding at very high salt 
concentration ${ }^{32}$ or PEG-to-PEG-pushing, ${ }^{5,6,33}$ equilibrium PEG partitioning in aHL (and possibly in other channels) is to a good extent regulated by the above dielectrophoretic and hydrostatic forces. To this end we model the polymer as a (low permittivity) spherical, neutral object immersed in an ionic solution of given ionic strength and calculate the change in the polymer self-energy when entering the pore. The initial motivation of this study was the reported experimental evidence that the charge state of aHL channel modifies neutral PEG partitioning into the pore in a molecular weight (MW) dependent way. ${ }^{2}$ Here we demonstrate that the difference in free energy of PEG partitioning at two different $\mathrm{pH}$ conditions can be quantitatively accounted by assuming PEG is a dielectric spherical object that feels the dielectrophoretic and hydrostatic repulsive forces originated in the protein ionizable residues lining the pore lumen. We also show that the solvent electroosmotic flow induced by ion current is expected to have a negligible effect on PEG equilibrium partitioning. Notwithstanding the limitations of the model, the agreement found with PEG partitioning free energies determined from single-channel conductance experiments seems remarkable, considering the use of only PEG MW-dependent hydrodynamic radius and aHL structural data without fitting parameters. These results might help to improve the analysis of nanopore sizing based on PEG partitioning and conductance measurements by considering the influence of nanopore charges on neutral polymers.

\section{Materials and Methods}

\section{Basic equations}

We consider a neutral particle with permittivity $\varepsilon_{p}$ immersed in an electrolyte solution with permittivity $\varepsilon_{w}$ and Debye length $\kappa^{-1}$. We denote by $\phi(\mathbf{r})$ and $\mathbf{E}(\mathbf{r})$ the electric potential and electric field, respectively, created by a charged interface at the point $r(x, y, z)$. The dielectrophoretic force acting on the particle can be obtained by integrating the Maxwell stress tensor over the particle surface $S_{p}$. Generally, the electrostatic force on a surface $S_{p}$ in a medium of permittivity $\varepsilon$ is: ${ }^{27,34,35}$

$$
\mathbf{F}_{e}=\varepsilon \int_{S_{p}}\left(\mathbf{E}(\mathbf{r})[\mathbf{E}(\mathbf{r})]^{\mathrm{T}}-\frac{1}{2}|\mathbf{E}(\mathbf{r})|^{2} \mathbf{I}\right) \mathbf{n}(\mathbf{r}) d S
$$

where $\mathbf{I}$ is the identity matrix, $\mathbf{n}(\mathbf{r})$ is the unit normal vector pointing away from the surface $S_{p}$ and superindex $T$ indicates a transposed matrix. The electric field $\mathbf{E}(\mathbf{r})$ can only be found in closed form for a few particular cases (planar geometry, absence of ions in solution, etc.). The force acting on the fluid surrounding the particle, here denoted as hydrostatic force, $\mathbf{F}_{H}$, can be obtained from integration of the pressure tensor ${ }^{27}$ over the particle surface: 


$$
\mathbf{F}_{H}=\int_{S_{p}}\left(-p(\mathbf{r})+\eta \nabla \mathbf{u}(\mathbf{r})+\eta[\nabla \mathbf{u}(\mathbf{r})]^{\mathrm{T}}\right) \mathbf{n}(\mathbf{r}) d S
$$

where $\eta$ stands for the viscosity of the fluid and $p(\mathbf{r})$ for the local pressure. For steady-state conditions, mass conservation leads to zero divergence of the solvent velocity $\mathbf{u}(\mathbf{r})$ and eq 2 simplifies to:

$$
\mathbf{F}_{H}=-\int_{S_{p}} p(\mathbf{r}) \mathbf{n}(\mathbf{r}) d S
$$

The pressure $p(\mathbf{r})$ follows from solving the Navier-Stokes equation, which in our case simplifies to the form:

$$
\nabla p(\mathbf{r})-\rho(\mathbf{r}) \mathbf{E}(\mathbf{r})=0
$$

where $\rho(\mathbf{r})$ is the local charge density due to the mobile ions in solution. Before attempting the integration of eqs 1 and 3 to get the total force on the particle $\mathbf{F}=\mathbf{F}_{D}+\mathbf{F}_{H}$, one needs to solve the Poisson-Boltzmann equation for the local electric field created by the channel charges:

$$
\nabla^{2} \phi(\mathbf{r})=\frac{k_{B} T}{e} \kappa^{2} \sinh \left(\frac{e}{k_{b} T} \phi(\mathbf{r})\right)
$$

where $k_{B}$ is Boltzmann constant, $T$ the absolute temperature and $e$ the elementary charge. Note that eq 5 is written for a 1:1 electrolyte for the sake of simplicity.

\section{Numerical Procedure}

We used the 3D atomic structure of the channel available at the Protein Data Bank (access code 7AHL) for the numerical calculation of the electrophoretic and hydrostatic forces on PEG solutes in the aHL channel. Then, two structures differing in the ionization state of some residues (corresponding to solution $\mathrm{pH} 4.5$ and 7.5) were obtained by using the PROPKA algorithm implemented in pdb2pqr (version 2.1.1.). ${ }^{36}$ This tool yields a PQR file, a format similar to PDB with additional information about the radii and charge of each atom at the selected $\mathrm{pH}$ (in our case we used the CHARMM force field included in the application). The PQR files corresponding to each $\mathrm{pH}$ were used to calculate the electrostatic potential under conditions mimicking the protein inserted in a lipid membrane. The code used here is a modification of the one used previously ${ }^{37,38}$ for solving the Poisson-Nernst-Planck equations. The same code can be used (although with a somewhat higher computational cost) to solve the linear or nonlinear PoissonBoltzmann (PB) equation (by imposing zero applied potential and zero current conditions). 
The numerical solution of the PB equation yields a 3D map of the electric potential $\phi(\mathbf{r})$ and the field $\mathbf{E}(\mathbf{r})$ within the simulation box. These quantities are needed for the estimation of the dielectrophoretic and hydrostatic force (eqs 1 and 3) felt by the neutral particle in the integration regions. To get the dielectrophoretic force we use the expression due to Cai et al., ${ }^{39}$ obtained by direct integration of the Maxwell stress tensor (eq 1) over the boundary surface of the particle:

$$
\mathbf{F}_{D}=-\int_{S_{p}} \frac{1}{2}\left(\varepsilon_{w}-\varepsilon_{p}\right)\left(\mathbf{E}_{o} \mathbf{E}_{i}\right) \mathbf{n}(\mathbf{r}) d S
$$

Where the subscripts in $\mathbf{E}_{o}$ and $\mathbf{E}_{i}$ refer to the electric field outside $(o)$ and inside $(i)$ the particle boundary surface. For mathematical details of the derivation of eq 6 see e.g. the appendix of reference ${ }^{39}$. Two consequences follow from eq 6 : First, the dielectrophoretic force $\mathbf{F}_{D}$ cancels out when the dielectric permittivity of the particle equals that of the surrounding medium; Second, this force is symmetrical with respect the sign of the source charges, as the sign of both $\mathbf{E}_{o}$ and $\mathbf{E}_{i}$ will change simultaneously. Eq 6 can be further simplified using the following boundary conditions over the surface of the particle $(r=R)$ :

$$
\begin{aligned}
& \varepsilon_{w} E_{o \perp}=\varepsilon_{p} E_{i \perp} \\
& E_{o \|}=E_{i \|}
\end{aligned}
$$

in terms of the normal $(\perp)$ and parallel $(\|)$ components of the electric field outside or inside a particle of radius $R$. Using the outside electric field, we get for eq 6 :

$$
\mathbf{F}_{D}=-\int_{S_{p}} \frac{1}{2}\left(\varepsilon_{w}-\varepsilon_{p}\right)\left(E_{o \perp}{ }^{2} \frac{\varepsilon_{w}}{\varepsilon_{p}}+E_{o \|}{ }^{2}\right) \mathbf{n}(\mathbf{r}) d S
$$

Whereas from eqs 3 and 4 in the linear approximation for the potential we get for the hydrostatic force:

$$
\mathbf{F}_{H}=-\frac{\varepsilon_{w} \kappa^{2}}{2} \int_{S_{p}} \phi(\mathbf{r})^{2} \mathbf{n}(\mathbf{r}) d S
$$

The solution to the Poisson-Boltzmann, eq 5, and the computation of the eqs 8 and 9 was achieved by using FiPy, ${ }^{40}$ a partial differential equation solver written in Python. ${ }^{41}$ In our case, once the pqr structure was loaded into FiPy, we defined an unstructured tetrahedral mesh in the solution box around the protein and the model membrane (a low dielectric region mimicking the biological membrane), and also within a spherical surface enclosing the low dielectric particle (PEG model) with variable radius and position. This was accomplished by using gmsh ${ }^{42}$ from within the FiPy-Python environment. Additional code developed for this purpose allows the reading and processing of the three-dimensional structures of the channel protein in PQR format. The protein structure was oriented with the channel axis parallel to the z-axis. Subsequently, a 
region inaccessible to the solution was added to represent the membrane, thus dividing the box into two regions connected by the protein channel. The result is that the integration box comprises three regions with different dielectric constants and ionic and solvent accessibility: The protein/membrane, the solution, and the spherical particle. Since FiPy does not allow internal boundary conditions to be applied, ionic accessibility, solvent-accessible regions and other position-dependent quantities are constructed using a mask, so that internal boundary conditions are automatically satisfied. Regarding the boundary conditions on the external surfaces of the integration box, FiPy by default establishes no flows and/or fields in all of them (Neumann type). Therefore, it is only necessary to assign specific potential values (Dirichlet type) on external surfaces perpendicular to the axis of the channel (i.e. at $z= \pm L$ ) that can be identified with the values of the electric potential on the bulk solution on both sides of the membrane (zero in this work). FiPy uses PySparse by default, ${ }^{43}$ which in our tests proved to be extraordinarily stable and robust in terms of convergence under different conditions and geometries. PB equation is solved iteratively until convergence at the desired accuracy is reached (when the residue - the result of solving with the sweep order in FiPy - of the corresponding equation is less than $5 \cdot 10^{-7}$ ). In all cases an unstructured tetrahedral mesh of characteristic size $1.5 \AA$ has been used all over the integration box. All calculations were carried out on an Intel-i7 12-core workstation with $140 \mathrm{~GB}$ of RAM.

\section{Results and Discussion}

The aHL toxin forms heptameric pores in lipid bilayers. The transmembrane part of the pore, also known as the stem part, is nearly cylindrical. The bulky part of the channel is the cap domain, which has a large cavity with a narrow entrance (Figure 1). Each one of the seven subunits contains 293 amino acids, of which 95 are ionizable. Their ionization state at neutral pH makes the channel slightly anion selective because of its net positive charge. Negative and positive charges display axial symmetry, but they are asymmetrically distributed along the pore. We assume that the change from $\mathrm{pH} 7.5$ to $\mathrm{pH} 4.5$ does not induce conformational changes in the pore. ${ }^{44-46}$ From the spatial distribution of ionizable residues we calculated the protonation state of each amino acid, which is influenced by the neighbor residues, ${ }^{45}$ as detailed in the Methods section. Then, the electric potential and the electric field over the pore and the surrounding solution was mapped.

Bezrukov and Kasianowicz ${ }^{2}$ measured the free energy change of a single PEG molecule partitioning into aHL channel caused by channel side chain titration (from $\mathrm{pH} 7.5$ to $\mathrm{pH} 4.5$ ). They performed single conductance experiments with $15 \%(\mathrm{w} / \mathrm{w})$ of a given MW PEG added symmetrically to $1 \mathrm{M} \mathrm{NaCl}$ solutions. Whereas in absence of PEG channel conductance increased 
by $15 \%$ upon lowering three $\mathrm{pH}$ units, in PEG solutions the same $\mathrm{pH}$ change decreased the apparent cut-off size of PEG entry. The change $\Delta \Delta G\left(\equiv \Delta G_{\mathrm{pH} 4.5}-\Delta G_{\mathrm{pH7.5}}\right)$ in polymer free energy of partitioning induced by channel titration was a small and positive quantity and it scaled with the square of the polymer MW. Therefore, as the channel net positive charge was increased, the PEG cut-off size to penetrate the aHL pore became smaller. Note that lowering the solution $\mathrm{pH}$ from 7.5 to 4.5 increases by more than $50 \%$ the channel anionic selectivity (permeability ratio varies from $\mathrm{P}_{-} / \mathrm{P}_{+}=2.7$ to 4.3 in $\left.0.3 / 0.1 \mathrm{M} \mathrm{KCl}\right) .{ }^{47}$
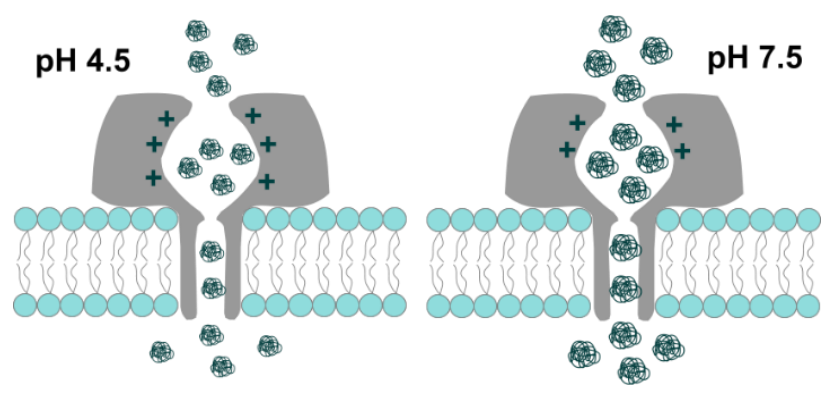

Figure 1. Cartoon that illustrates the effect of channel charge on the partitioning of neutral PEG molecules into the aHL channel. Channel (net positive) charge is higher at $\mathrm{pH} 4.5$ than at $\mathrm{pH}$ 7.5. The channel-PEG interaction is seen in a reduction of the cut-off size of the biggest PEG able to enter the pore from $\mathrm{pH} 7.5$ to $\mathrm{pH} 4.5$.

They speculated about the origin of this unusual "net charge effect" on a neutral polymer. Among possible causes they mentioned the change in water properties induced by protonation of some amino acid side chains and the polymer exclusion near charged sites because of their lower dielectric constant than water. We attempt here to explain quantitatively the increase in PEG free energy of confinement observed upon titration of aHL channel by calculating the repulsion of a low permittivity neutral particle immersed in an electrolyte solution near a charged surface. We model a PEG molecule as a neutral, low permittivity sphere with the same radius as the characteristic hydrodynamic radius of each PEG according to its MW and assume that PEG dielectric constant is ca. $10 .{ }^{48,49}$ However, our calculations showed that the actual value of this parameter has little influence in the total force acting on the particle, provided PEG dielectric constant is much smaller than that of the salt solution (ca. 78-80). No significant differences were found by using for $\varepsilon_{p}$ values 6,10 or 20 times the vacuum permittivity.

From numerical integration of the dielectrophoretic and the hydrostatic force, eqs 8 and 9, we obtain the total force $\mathbf{F}$ ( $\equiv \mathbf{F}_{\mathrm{D}}+\mathbf{F}_{\mathrm{H}}$ ) acting on a neutral particle of radius $R$ when it approaches the 
channel opening along the $z$ axis of the protein. This is the most energetically favorable particle path given the axial symmetry of this heptameric channel. In addition, the number of PEG molecules within the cap side of the channel is very small in a 15\% PEG solution (between 5 and 1 for PEGs of 600-2000 Da). Therefore, we can assume as a first approximation that PEG partitioning occurs in single-file fashion. By integrating this repulsive force $\mathbf{F}$ (from a virtual infinite distance up to a given location) we calculate the characteristic self-energy of the particle at each axial position. This energy is strongly dependent on the relative size of the particle with respect the pore opening as well as on the pore charge.

Figure 2 illustrates this dependence with calculations of the self-energy of the particle that moves along the symmetry axis of the aHL channel towards its cap entrance (The particle self-energy in the stem entrance is much less significant for reasons that will be mentioned later). The highest energy is achieved at the transition between the entrance funnel and the cavity, where the particle gets nearer the protein charges. Fig. $2 \mathrm{~A}$ shows that a small change in the particle size (from 7.5 to $9 \AA$ in radius) is enough to increase five times the energy peak at pH 7.5. Figure 2B shows the self-energy for a $7.5 \AA$ particle for two different charge states of the aHL channel, corresponding to $\mathrm{pH} 7.5$ and $\mathrm{pH}$ 4.5. The increase in net positive charge caused by (possibly partial) protonation of some residues with effective pKa in the range 4.5-7.5 yields stronger dielectrophoretic and hydrostatic force on the particle.
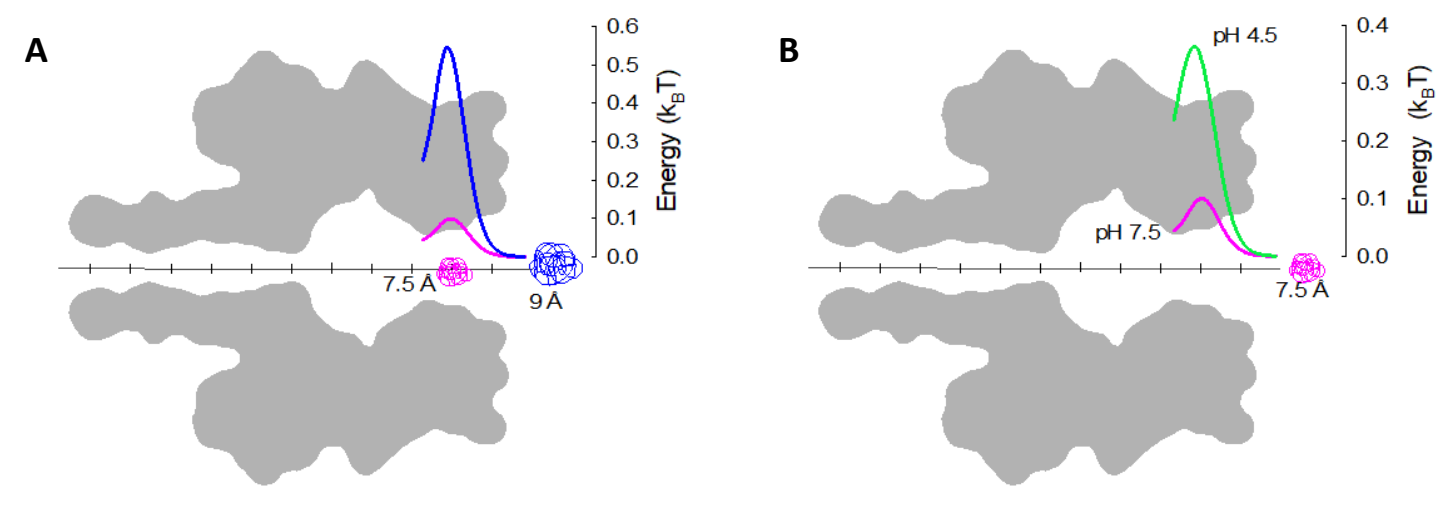

Figure 2. Self-energy of a spherical particle partitioning between the external solution and the cap side (entrance radius $\sim 13 \AA$ ) of the aHL pore in a $1 \mathrm{M} \mathrm{KCl}$ solution. A) The energy peak is highly sensitive to the size of the particle (radii 7.5 and $9 \AA$ as depicted). B) Self-energy of a particle with radius $7.5 \AA$ in solutions of pH 7.5 and 4.5 as labeled. Protonation of a few amino acids increases the net positive charge in the pore and, consequently the energy penalty for partitioning into the pore. A dashed line with $1 \mathrm{~nm}$ spaced marks is depicted along the 
longitudinal axis of the channel to help as reference for the position of the peak. The shaded region is a cross section of the protein aHL (slightly edited but keeping the original aspect ratio).

The relative contribution of the dielectrophoretic force $F_{D}$ and the hydrostatic force $\mathbf{F}_{\mathrm{H}}$ to the energy also depends on the size of the particle and the protein charge. Figure 3 shows the corresponding energies $E_{D}$ and $E_{H}$ (of the particle on the cap side) for three particle radii and two protein charge states corresponding to $\mathrm{pH} 7.5$ and $\mathrm{pH} 4.5$. For low pore charge density $(\mathrm{pH} 7.5)$ the two energy profiles slightly differ in the position of their peak, whereas such difference becomes negligible at $\mathrm{pH}$ 4.5. The superposition or relative displacement between maxima of the two curves is probably related to the fact that $F_{D}$ varies with the square of the electric field (see eq. 8) while $\mathbf{F}_{H}$ varies with the square of the electric potential (see eq. 9). On top of that there is an additional integration over the particle surface which "disconnects" the final numerical result from local values of the electric potential and the field. For particle sizes much smaller than the channel aperture the peak energy associated to the hydrostatic force is several times greater than the one originated by the dielectrophoretic force. This is shown in the two bottom plots of Figure $4(R=7 \AA$ at $\mathrm{pH} 7.5$ and $R=9 \AA$ at $\mathrm{pH} 4.5)$. However, for particle radii closer to that of the channel aperture (radius $\sim 13 \AA$ ), the opposite happens and $E_{D}$ attains higher values than $E_{H}$, as shown in the two top plots of Figure $4(R=10 \AA$ at $\mathrm{pH} 7.5$ and $R=10.5 \AA$ at $\mathrm{pH} 4.5)$. This transition between prevailing $E_{H}$ to prevailing $E_{D}$ occurs at both $\mathrm{pH}$. This effect is possibly related to the peculiar geometry and charge distribution of the aHL channel entrance. In addition, this relative contribution may suggest that other calculations for a model nanopore-particle idealized system 35 that yielded hydrostatic forces much greater than dielectrophoretic ones cannot be extrapolated to protein channels. 
$\mathrm{pH} 7.5$
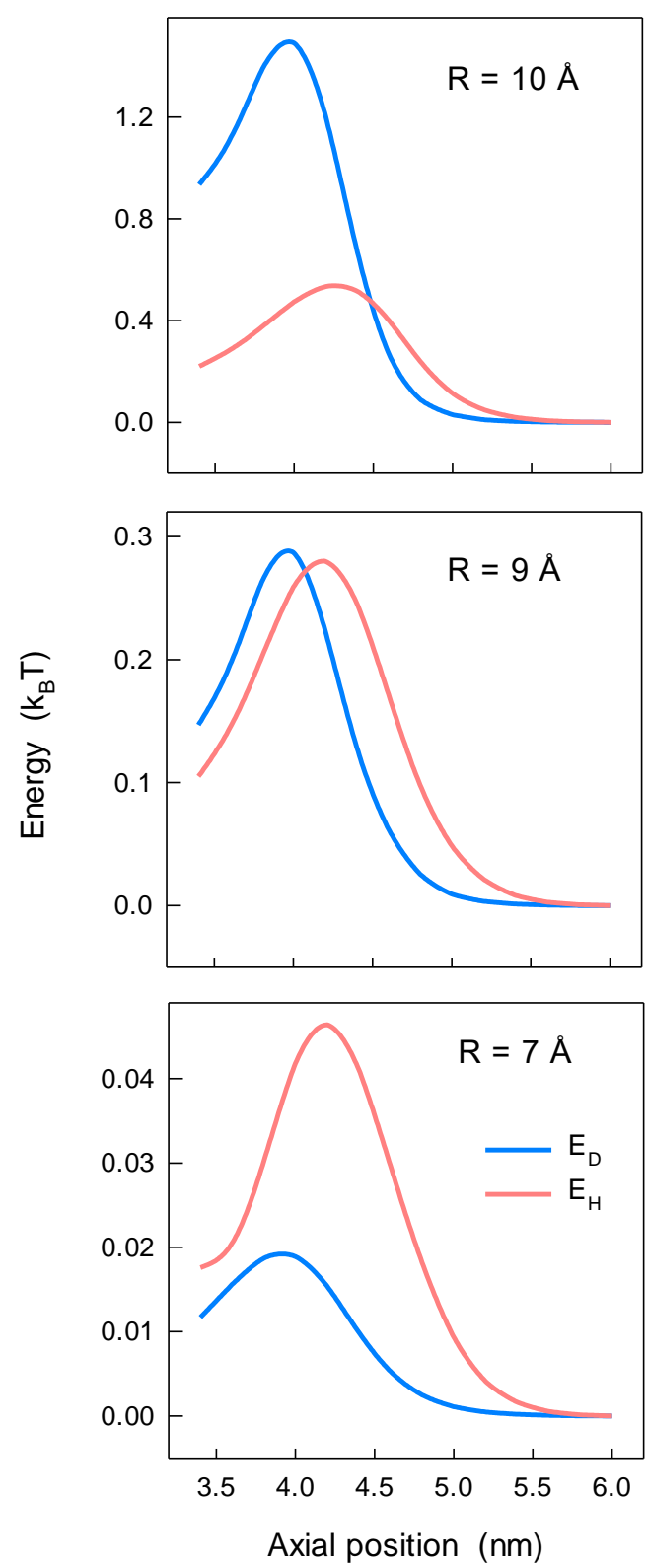

$\mathrm{pH} 4.5$
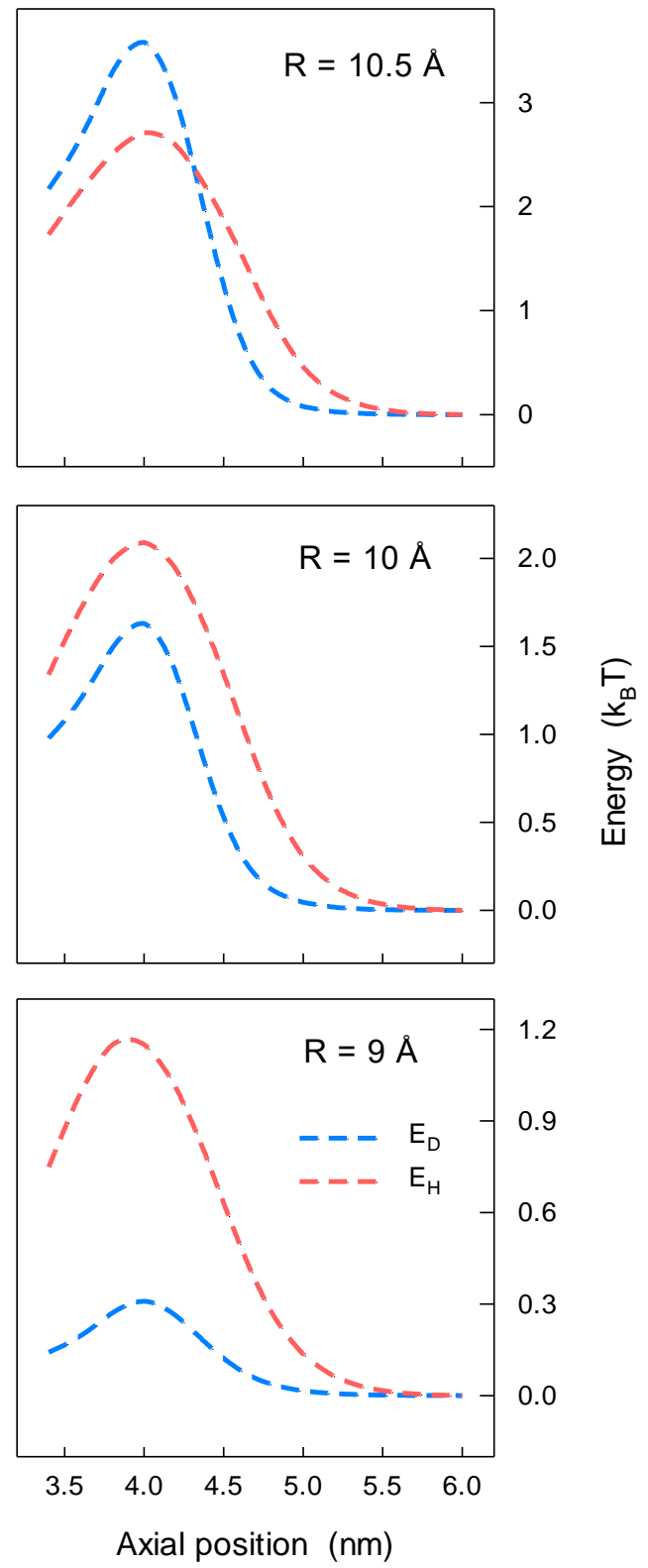

Figure 3. Self-energy of spherical particles of different radii (as labeled) partitioning between solution and the cap side of the aHL pore in a $1 \mathrm{M}$ solution of a monovalent salt at $\mathrm{pH} 7.5$ (left panel, solid lines) and $\mathrm{pH} 4.5$ (right panel, dashed lines). The energy $E_{D}$ corresponding to dielectrophoretic force and the energy $E_{H}$ corresponding to hydrostatic force are represented by blue and pink curves, respectively. The zero of the axial coordinates corresponds to the center of the longitudinal axis of the protein channel (the protein spans approximately from -5 to $+5 \mathrm{~nm}$ ). 
Top, middle, and bottom panel corresponds to charge state and particle radius combinations that yield $E_{D}>E_{H}, E_{D} \approx E_{H}$ and $E_{D}<E_{H}$, respectively.

Figure 4 displays calculations of the particle self-energy averaged over the cap entrance region (where ionization of some residues might change the total force acting on the particle) for $\mathrm{pH} 4.5$ (blue squares) and pH 7.5 (green circles).

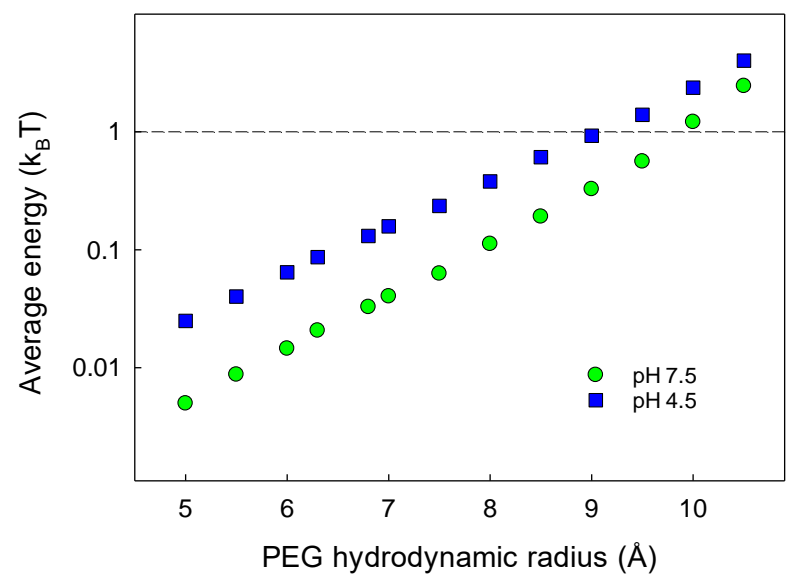

Figure 4. Average self-energy of a spherical particle partitioning between solution and the cap side of the $\mathrm{aHL}$ pore in a $1 \mathrm{M}$ solution of a monovalent salt. Blue squares correspond to calculations at $\mathrm{pH} 4.5$ and green circles to $\mathrm{pH}$ 7.5. A dashed line is drawn for an energy comparable to the thermal energy of the particle.

An increase in particle radius from 5 to $10 \AA$ yields two orders of magnitude increase in the average self-energy. The horizontal dashed line for an energy of $1 k_{B} T$ is an aid to visualize the size of a particle having thermal energy comparable to its average self-energy when entering the aHL channel through the cap aperture at two different $\mathrm{pH}$. A particle of radius $8.5 \AA$ at $\mathrm{pH} 4.5$ and a particle of $9.5 \AA$ at $\mathrm{pH} 7.5$ have the same energy. This means that, at least qualitatively, the dielectrophoretic and the hydrostatic force might explain the different PEG partitioning into aHL channel observed upon titration.

As expected, the channel charge screening modulates the interaction between the pore and the particle. The salt concentration in the solution plays an important role in the energy associated to the dielectrophoretic and hydrostatic repulsive forces on the particle. Both the increase of the size of the electric double layer (as ionic strength is lowered) and the increase in pore charge (concomitant with $\mathrm{pH}$ decrease) contribute to enhance the particle repulsion and the corresponding average energy of the particle in the cap entrance. Figure 5 illustrates this effect for the two charge states of the aHL channel considered so far and a neutral particle of radius 
$7.4 \AA$ (approximately half the radius of the cap entrance). At $\mathrm{pH} 4.5$ the change in the average energy of the particle in the cap entrance from $1 \mathrm{M}$ up to physiological concentration is almost an order of magnitude. At $\mathrm{pH} 7.5$ the energy doubles its value from $1 \mathrm{M}$ to $0.01 \mathrm{M}$.

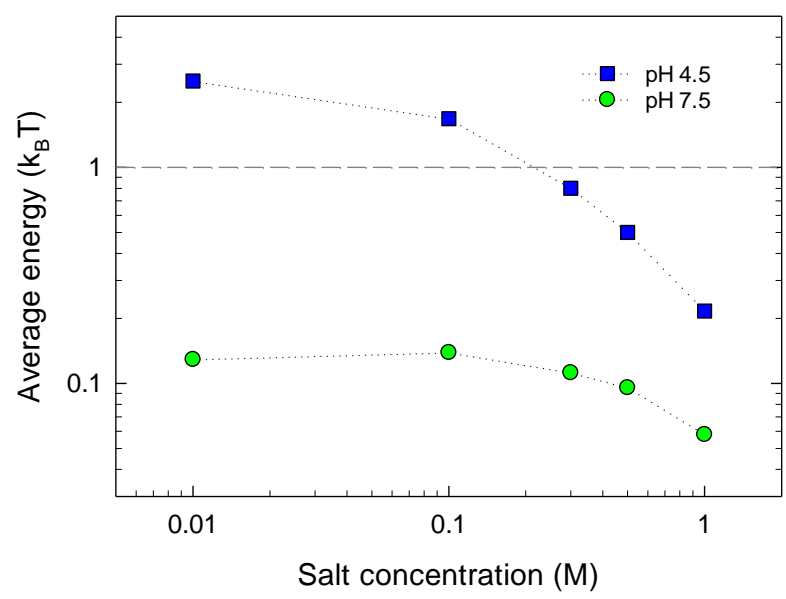

Figure 5. Average self-energy of a spherical particle of radius $7.4 \AA$ partitioning between solution and the cap side of the aHL pore in solutions of monovalent salts of several concentrations. Blue squares correspond to calculations at $\mathrm{pH} 4.5$ and green circles to $\mathrm{pH}$ 7.5. A dashed line is drawn for an energy comparable to the thermal energy of the particle.

The above model calculations of the dielectrophoretic and hydrostatic energy of neutral, dielectric particles of varying size involve no extra fitting parameters. Numerical solution of eqs 8 and 9 for the spatial charge distribution and geometry of the cap aperture of aHL channel (from its 3D atomic structure) yield average energy values similar to the thermal energy and highly sensitive to the protein charge state and to the particle size. Our next step is comparing the computed energies with partitioning free energy estimates from single channel conductance measurements. This involves the assumption, already mentioned in the Introduction, of considering a PEG molecule as a neutral spherical particle. We assume that the free energy of partitioning $\triangle G$ of a PEG molecule between bulk solution and the channel (under equilibrium conditions) includes an energetic contribution $\Delta E$ (which may be negative or positive depending on whether the prevailing interaction with the channel is attractive or repulsive, respectively) and a positive term, $-T \Delta S$, accounting for the entropic loss associated to PEG chains confinement in the pore:

$$
\Delta G=\Delta E-T \Delta S
$$


In the absence of any PEG-to-PEG interaction in bulk solution (dilute regime) PEG entry will be observed when $\Delta G$ is much smaller or comparable to thermal energy $k_{B} T$. To circumvent the difficult estimation of the entropic loss $\Delta S$, we calculate the change in $\Delta G$ between two partitioning equilibria at different $\mathrm{pH}$, with presumably identical $\Delta S$. Therefore, we hypothesize that $\Delta \Delta G=\Delta \Delta E$. Table 1 shows estimates of $\Delta \Delta G_{\text {exp }}$ from channel conductance measurements ${ }^{2}$ and calculations of the change in the average energy $\Delta \Delta E_{\text {calc }}$ for a model particle having the same radius as the PEG molecule. We consider only PEG molecules small enough to enter the channel through the cap side (up to MW 2000 approximately).

\section{Table 1}

pH-induced shift in PEG free energy of partitioning and calculated average energy between two channel charge states

\begin{tabular}{|c|c|c|c|c|}
\hline $\begin{array}{l}\text { PEG MW } \\
\text { (Da) }\end{array}$ & $\begin{array}{l}\text { Hydrodynamic } \\
\text { radius* }(\AA)\end{array}$ & $\begin{array}{c}\Delta \Delta G_{\exp }\left(k_{B} T\right) \\
(p H 7.5 \rightarrow p H 4.5)\end{array}$ & $\begin{array}{c}\Delta \Delta \mathrm{E}_{\mathrm{calc}}\left(\mathrm{k}_{\mathrm{B}} \mathrm{T}\right) \\
(\mathrm{pH} 7.5 \rightarrow \mathrm{pH} 4.5)\end{array}$ & Effective radius ${ }^{\#}(\AA)$ \\
\hline 600 & 5.7 & 0.07 & 0.04 & 6.4 \\
\hline 1000 & 7.4 & 0.21 & 0.16 & 7.7 \\
\hline 1540 & 9.3 & 0.51 & 0.74 & 8.8 \\
\hline 2000 & 10.6 & 0.83 & 1.64 & 9.5 \\
\hline
\end{tabular}

* Estimated following 50

\# Radius of the sphere that yields $\Delta \Delta \mathrm{E}_{\mathrm{calc}}=\Delta \Delta \mathrm{G}_{\mathrm{exp}}$

The effective radius shown in Table 1 is the radius of the particle needed to yield $\Delta \Delta E_{\text {calc }}=\Delta \Delta G_{\exp }$. It is very close to the PEG hydrodynamic radius (differences are ca $10 \%$ or less). It is reasonable to think that large polymers whose characteristic size is closer to the pore aperture must suffer a larger deformation to enter the pore. The assumption of PEG as a spherical object becomes questionable in this range. Note also that the theoretical and experimental determination of the PEG hydrodynamic radius is not without difficulties. There are small differences in the values of the scaling coefficient $v$ of the relationship $R_{h} \sim(\mathrm{MW})^{v}$ reported in the literature between PEG hydrodynamic radius and MW. Depending on the experimental technique used (viscosimetry, diffusion, Small Angle Neutron Scattering, Dynamic Light Scattering) $v$ ranges from 0.4 to 0.57 (Classical scaling theory predicts $0.55-0.57^{23}$ ). Here we follow the values reported by Lee et al. ${ }^{50}$ for unhydrated PEGs that take into account the shape anisotropy of diffusing PEGs through a pore. 
Interestingly, the agreement between calculations and experiments is good for low MW PEGs whereas becomes poor for larger polymers. Still, calculations capture the order of magnitude of the change in partitioning free energy $\Delta \Delta G_{\text {exp }}$ between $\mathrm{pH} 7.5$ and $\mathrm{pH} 4.5$. Figure 6 shows additional calculations of $\Delta \Delta E_{\text {calc }}$ for several radii of PEGs superimposed with free energy change obtained from experiments ${ }^{2}$.

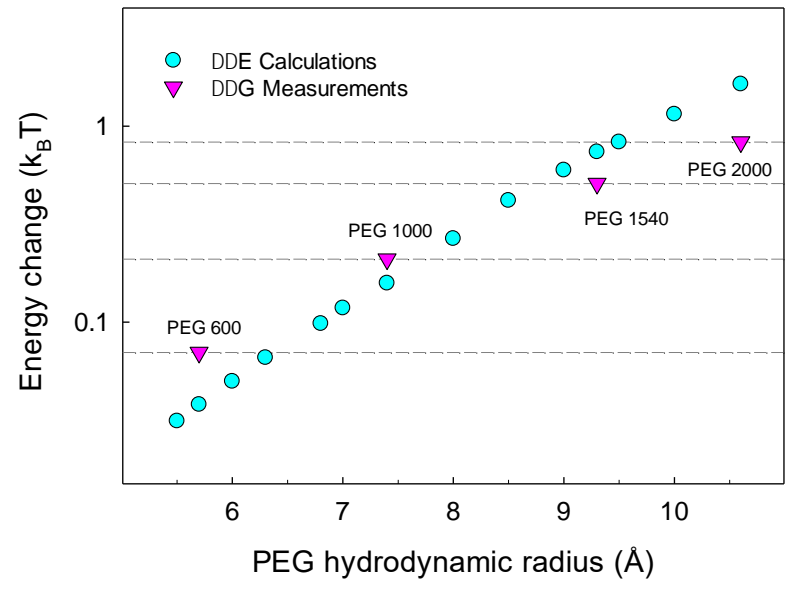

Figure 6. Change in the free energy of PEG partitioning between $\mathrm{pH} 7.5$ and $\mathrm{pH} 4.5, \Delta \Delta G_{\exp }$ (pink triangles) estimated from single-channel conductance measurements ${ }^{2}$ and calculations of the corresponding change in the average energy $\Delta \Delta E_{\text {calc }}$ (cyan circles) for a PEG molecule partitioning between solution and the cap side of the aHL pore in a $1 \mathrm{M}$ solution of a monovalent salt. In calculations, a PEG molecule is idealized as a spherical particle of radius $R_{\mathrm{h}}$. In the plot of $\Delta \Delta G_{\text {exp }}$ the hydrodynamic radius of PEG for each MW has been assigned following Lee et al. ${ }^{50}$.

The conductance experiments reported by Bezrukov and Kasianowicz ${ }^{2}$ were performed using symmetrical $15 \%$ PEG solutions on both sides of the channel. Therefore, we also calculated the corresponding change in average energy $\Delta \Delta E_{\text {calc }}$ for a PEG molecule entering the channel through the stem side. Because of the peculiar distribution of ionizable residues near this entrance, titration from $\mathrm{pH} 7.5$ to $\mathrm{pH} 4.5$ barely changes the net charge near this stem aperture. ${ }^{46}$ Consequently, the shift $\Delta \Delta E_{\text {calc }}$ on this side was more than an order of magnitude lower than the corresponding for the cap side, irrespective of the particle size. Interestingly, this change $\Delta \Delta E_{c a l c}$ was negative, i.e. a slightly smaller average energy for $\mathrm{pH} 4.5$ than for $\mathrm{pH} 7.5$. This result is consistent with the fact that the aHL channel exhibits a small net negative charge near the stem mouth ${ }^{30}$ and partial titration of some aspartic or glutamic residues decreases the net charge (opposite to what happens on the cap entrance where net positive charge increases upon lowering $\mathrm{pH}$ ). The dielectrophoretic and the hydrostatic forces between a neutral PEG molecule and a charged surface depend on the square of the electric field and the square of the electric 
potential, respectively (see Methods section). Then, a decrease in negative charge implies a decrease in the energy. For PEG 1540 and PEG 2000 we get $\Delta \Delta E_{\text {calc }}=-0.06 k_{B} T$ and $\Delta \Delta E_{\text {calc }}=-0.13$ $k_{B} T$, respectively. These values are much lower in magnitude than the corresponding ones in Table 1 for the cap side and too small to compensate for the overestimation seen in the $\Delta \Delta E_{\text {calc }}$ values for these large PEGs.

One may ask whether the model accurately captures the free energy of partition $\Delta G_{\text {exp }}$ rather than the difference $\Delta \Delta G_{\text {exp }}$ between two different channel charge states. Table 2 shows the free energy of PEG confinement $\Delta G_{\text {exp }}$ for each $\mathrm{pH}$ (calculated from data reported by Bezrukov et al. ${ }^{2}$ ) as well as the calculated average energy $\Delta E_{\text {calc }}$ for the same conditions. For the small PEG 600 and PEG1000 in both $\mathrm{pH}$ conditions, $\Delta E_{c a l c}<\Delta G_{\text {exp }}$ and their values do not differ much. This is consistent with the fact that the entropic penalty for partitioning of polymers much smaller in size than the channel aperture should be very low and hence $\Delta G \approx \Delta E$. In contrast, for PEG1540 and PEG2000 the calculated energy and the free energy obtained from conductance measurements differ by factors of $\sim 1.3-4$. Interestingly, $\Delta G_{\text {exp }}<\Delta E_{\text {calc. }}$. We would expect right the opposite, given that the entropic contribution to the free energy of confinement (-TAS) is positive and adds up to the energy term $\Delta E$. We speculate that the suspected attractive interaction between PEG molecules and hydrophobic segments of the channel previously reported, ${ }^{1}$ which is overlooked in our model, could be an explanation for this overestimation of the free energy of confinement. However, we do not have a satisfactory explanation of such effect. Most probably, modelling the PEG molecule as a sphere with a radius like the PEG hydrodynamic radius in free solution and ignoring their possible deformation upon pore entry becomes questionable when the radius is very close to the aperture size. This would lead to an overestimation of the dielectrophoretic and hydrostatic forces and hence to unrealistic values of $\Delta E_{\text {calc }}$ because this calculated average energy increases very steeply with the PEG radius for large PEGs.

Table 2

PEG free energy of partitioning and calculated average energy for both channel charge states

\begin{tabular}{|c|c|c|c|c|}
\hline \multirow{2}{*}{$\begin{array}{c}\text { PEG MW } \\
\text { (Da) }\end{array}$} & \multicolumn{2}{|c|}{ pH 7.5 } & \multicolumn{2}{c|}{ pH 4.5 } \\
\cline { 2 - 5 } & $\Delta \mathbf{G}_{\exp }\left(\mathrm{k}_{\mathrm{B}} \mathrm{T}\right)$ & $\Delta \mathrm{E}_{\text {calc }}\left(\mathrm{k}_{\mathrm{B}} \mathrm{T}\right)$ & $\Delta \mathrm{G}_{\text {exp }}\left(\mathrm{k}_{\mathrm{B}} \mathrm{T}\right)$ & $\Delta \mathrm{E}_{\text {calc }}\left(\mathrm{k}_{\mathrm{B}} \mathrm{T}\right)$ \\
\hline 600 & 0.017 & 0.011 & 0.11 & 0.05 \\
\hline 1000 & 0.089 & 0.058 & 0.34 & 0.22 \\
\hline 1540 & 0.29 & 0.45 & 0.90 & 1.19 \\
\hline 2000 & 0.78 & 2.91 & 1.59 & 4.4 \\
\hline
\end{tabular}


We estimated the possible effect of electroosmotic flow (EOF) on PEG partitioning, as EOF might occur in $\mathrm{NaCl}$ conductance measurements under an applied voltage ${ }^{2}$ and it is sensitive to the channel charge. In fact, under positive applied voltage, there would be an EOF towards the cis side, partially hindering the polymer entry into the cap side of the channel. Therefore, the solvent drag on the PEG would be added to the repulsive dielectrophoretic and hydrostatic force acting on it. The EOF in aHL channel was demonstrated on two different studies ${ }^{51,52}$ using the neutral molecule beta-cyclodextrin (similar in size to PEG 1000). By using values of aHL selectivity ${ }^{51}$ and conductance ${ }^{2}$ measured in $1 \mathrm{M} \mathrm{NaCl}$ solutions at $\mathrm{pH} 7.5$ and $\mathrm{pH} 4.5$ we estimated the drag force on PEGs, which is typically an order of magnitude lower than the sum of the dielectrophoretic and hydrostatic force. For a particle of $7.4 \AA$ like PEG 1000, this drag force is about $0.03 \mathrm{pN}$ whereas $\mathrm{F}_{\mathrm{D}}+\mathrm{F}_{\mathrm{H}} \approx 0.3 \mathrm{pN}$. Therefore, under the typical voltage (100 $\mathrm{mV}$ ) and high salt concentration of the ionic conductance measurements ${ }^{2}$ used to compare with our calculations, the effect of the EOF on PEG partitioning in aHL is expected to be negligible.

\section{Conclusions}

We have studied the equilibrium partitioning of PEG polymers with varying MW into a biological nanopore, the aHL toxin, for different $\mathrm{pH}$-induced charge states of this protein channel. The reported change in the ability of neutral polymers to enter the pore upon channel titration was analyzed. We have shown that the electric field created by the net positive charge of this nanopore induces two repulsive forces acting on the PEG molecule. First, a dielectrophoretic force $\mathbf{F}_{\mathrm{D}}$ resulting from the action of the electric field on a low polarizability particle like PEG immersed in a medium of higher dielectric constant. Second, a hydrostatic force $\mathbf{F}_{H}$ that pushes the PEG molecule away from the electric double layer to keep the right balance of concentration of mobile ions near the charged interface. General expressions for $\mathbf{F}_{\mathrm{D}}$ and $\mathbf{F}_{\mathrm{H}}$ have been derived from the Maxwell stress tensor and the pressure tensor, respectively. These forces and the corresponding PEG molecule self-energy have been numerically computed by using the 3D atomic structure of the $\mathrm{aHL}$ channel and its 3D charged residue spatial distribution for each $\mathrm{pH}$. The computation of the total self-energy of a PEG molecule that enters through each one of the channel openings and its averaged value over the cap and the stem entrance allowed its comparison with the free energy of PEG partitioning into aHL pore obtained from single channel conductance measurements. ${ }^{2}$ The computed energy increases with the PEG MW and the channel charge, in agreement with experiments. We found that the $\mathrm{pH}$-induced (from $\mathrm{pH} 7.5$ to $\mathrm{pH} 4.5$ ) shift in the measured free energy of partitioning $\Delta \Delta G_{\exp }$ is well reproduced by parameter-free calculations of the dielectrophoretic and hydrostatic forces based on the channel 3D structure. For low MW PEGs $\Delta \Delta E_{\text {calc }} \approx \Delta \Delta G_{\text {exp }}$, whereas calculations overestimate $\Delta \Delta G_{\text {exp }}$ for PEGs whose 
size is similar to the channel mouth, possibly because large PEG molecules no longer display a spherical shape to penetrate the nanopore and suffer some deformation. In the case of small polymers like PEG 600 and PEG 1000 the computed energy $\Delta E_{c a l c}$ is slightly lower than the partitioning free energy $\Delta G_{\text {exp }}$ both for experiments at $\mathrm{pH} 7.5$ and $\mathrm{pH} 4.5$, consistently with the fact that the entropic penalty for partitioning $(-T \Delta S)$ should be very small because of the relative size of the polymer and the channel aperture. For larger polymers $\Delta S$ is probably significant, and we find that $\Delta G_{\text {exp }}$ and $\Delta E_{c a l c}$ are different. Presently, we do not have a clear explanation for the unrealistic high values of $\Delta E_{\text {calc }}$ yielded by our calculations for larger polymers. The cause could be in misrepresenting the polymer as a sphere with the PEG hydrodynamic radius in bulk solutions. We also estimated the possible effect of electroosmotic flow on PEG partitioning and found that the solvent drag force on the PEG molecule can be neglected when compared with $\mathbf{F}_{\mathrm{D}}$ and $\mathbf{F}_{\mathrm{H}}$. Despite the limitations of the model, this is to our knowledge the first attempt to quantitatively evaluate of the energy involved in PEG partitioning into charged protein channels. Our approach shows that the interaction of large neutral solutes with charged nanopores should not be ignored even in solutions of high ionic strength where screening of pore charges is important. This charge effect fact is relevant to many nanopore-based sensors used in particle detection. We believe that these findings can be applied to other biological nanopores, so that the pore sizing experiments may be correctly analyzed by taking into account the effect of channel charge on neutral polymers. This study reports a new approach to accounting for the energy components of polymer partitioning into nanoscale pores, the phenomenon that underlies a great number of transport processes in biology.

\section{Acknowledgments}

This work was supported by the Government of Spain (project no. PID2019-108434GB-I00 AEI/FEDER, UE), and Universitat Jaume I (project no. UJI-B2018-53). The authors wish to dedicate this work to the memory of Dr. Philip A. Gurnev and pay homage to his fruitful career in polymer interaction with biological channels. 


\section{References}

(1) Bezrukov, S. M.; Vodyanoy, I.; Brutyan, R. A.; Kasianowicz, J. J. Dynamics and Free Energy of Polymers Partitioning into a Nanoscale Pore. Macromolecules 1996, 29 (26), 85178522.

(2) Bezrukov, S. M.; Kasianowicz, J. J. The Charge State of an Ion Channel Controls Neutral Polymer Entry into Its Pore. Eur. Biophys. J. 1997, 26 (6), 471-476.

(3) Gurnev, P. A.; Ortenberg, R.; Dörr, T.; Lewis, K.; Bezrukov, S. M. Persister-Promoting Bacterial Toxin TisB Produces Anion-Selective Pores in Planar Lipid Bilayers. FEBS Lett. 2012, 586 (16), 2529-2534.

(4) Niedzwiecki, D. J.; Mohammad, M. M.; Movileanu, L. Inspection of the Engineered FhuA $\Delta c / \Delta 4 L$ Protein Nanopore by Polymer Exclusion. Biophys. J. 2012, 103 (10), 2115-2124.

(5) Aksoyoglu, M. A.; Podgornik, R.; Bezrukov, S. M.; Gurnev, P. A.; Muthukumar, M.; Parsegian, V. A. Size-Dependent Forced PEG Partitioning into Channels: VDAC, OmpC, and $\alpha$-Hemolysin. Proc. Natl. Acad. Sci. U. S. A. 2016, 113 (32), 9003-9008.

(6) Gurnev, P. A.; Stanley, C. B.; Aksoyoglu, M. A.; Hong, K.; Parsegian, V. A.; Bezrukov, S. M. Poly(Ethylene Glycol)s in Semidilute Regime: Radius of Gyration in the Bulk and Partitioning into a Nanopore. Macromolecules 2017, 50 (6), 2477-2483.

(7) Krasilnikov, O. V.; Sabirov, R. Z.; Ternovsky, V. I.; Merzlyak, P. G.; Muratkhodjaev, J. N. A Simple Method for the Determination of the Pore Radius of Ion Channels in Planar Lipid Bilayer Membranes. FEMS Microbiol. Lett. 1992, 105 (1-3), 93-100.

(8) Bezrukov, S. M.; Vodyanoy, I. Probing Alamethicin Channels with Water-Soluble Polymers. Effect on Conductance of Channel States. Biophys. J. 1993, 64 (1), 16-25.

(9) Parsegian, V. A.; Bezrukov, S. M.; Vodyanoy, I. Watching Small Molecules Move: Interrogating Ionic Channels Using Neutral Solutes. Biosci. Rep. 1995, 15 (6), 503-514.

(10) Merzlyak, P. G.; Yuldasheva, L. N.; Rodrigues, C. G.; Carneiro, C. M. M.; Krasilnikov, O. V.; Bezrukov, S. M. Polymeric Nonelectrolytes to Probe Pore Geometry: Application to the $\alpha-$ Toxin Transmembrane Channel. Biophys. J. 1999, 77 (6), 3023-3033.

(11) Rostovtseva, T. K.; Nestorovich, E. M.; Bezrukov, S. M. Partitioning of Differently Sized Poly(Ethylene Glycol)s into OmpF Porin. Biophys. J. 2002, 82 (1), 160-169.

(12) Nestorovich, E. M.; Sugawara, E.; Nikaido, H.; Bezrukov, S. M. Pseudomonas Aeruginosa Porin OprF. Properties of the Channel. J. Biol. Chem. 2006, 281 (24), 16230-16237. 
(13) Ostroumova, O. S.; Gurnev, P. A.; Schagina, L. V.; Bezrukov, S. M. Asymmetry of Syringomycin E Channel Studied by Polymer Partitioning. FEBS Lett. 2007, 581 (5), 804808.

(14) Nablo, B. J.; Halverson, K. M.; Robertson, J. W. F.; Nguyen, T. L.; Panchal, R. G.; Gussio, R.; Bavari, S.; Krasilnikov, O. V.; Kasianowicz, J. J. Sizing the Bacillus Anthracis PA63 Channel with Nonelectrolyte Poly(Ethylene Glycols). Biophys. J. 2008, 95 (3), 1157-1164.

(15) Nestorovich, E. M.; Karginov, V. A.; Bezrukov, S. M. Polymer Partitioning and lon Selectivity Suggest Asymmetrical Shape for the Membrane Pore Formed by Epsilon Toxin. Biophys. J. 2010, 99 (3), 782-789.

(16) Gurnev, P. A.; Rostovtseva, T. K.; Bezrukov, S. M. Tubulin-Blocked State of VDAC Studied by Polymer and ATP Partitioning. FEBS Lett. 2011, 585 (14), 2363-2366.

(17) Movileanu, L.; Cheley, S.; Bayley, H. Partitioning of Individual Flexible Polymers into a Nanoscopic Protein Pore. Biophys. J. 2003, 85 (2), 897-910.

(18) Alcaraz, A.; López, M. L.; Queralt-Martín, M.; Aguilella, V. M. Ion Transport in Confined Geometries below the Nanoscale: Access Resistance Dominates Protein Channel Conductance in Diluted Solutions. ACS Nano 2017, 11 (10), 10392-10400.

(19) Zimmerberg, J.; Parsegian, V. A. Polymer Inaccessible Volume Changes during Opening and Closing of a Voltage-Dependent Ionic Channel. Nature 1986, 323 (6083), 36-39.

(20) Zimmerberg, J.; Bezanilla, F.; Parsegian, V. A. Solute Inaccessible Aqueous Volume Changes during Opening of the Potassium Channel of the Squid Giant Axon. Biophys. J. 1990, 57 (5), 1049-1064.

(21) Vodyanoy, I.; Bezrukov, S. M.; Parsegian, V. A. Probing Alamethicin Channels with WaterSoluble Polymers. Size-Modulated Osmotic Action. Biophys. J. 1993, 65 (5), 2097-2105.

(22) Flory, P. Principles of Polymer Chemistry; Cornell University Press: Ithaca N.Y., 1953.

(23) Gennes, P. G. de. Scaling Concepts in Polymer Physics; Cornell University Press, 1979.

(24) Pethig, R. Dielectrophoresis: Status of the Theory, Technology, and Applications. Biomicrofluidics 2010, 4 (2).

(25) Pohl, H. Dielectrophoresis: The Behavior of Neutral Matter in Nonuniform Electric Fields; Cambridge University Press: Cambridge ;New York, 1978.

(26) Parsegian, A. Energy of an Ion Crossing a Low Dielectric Membrane: Solutions to Four Relevant Electrostatic Problems. Nature 1969, 221 (5183), 844-846. 
(27) Masliyah, J. H.; Bhattacharjee, S. Electrokinetic and Colloid Transport Phenomena; John Wiley and Sons, 2005.

(28) Wang, H.; Ettedgui, J.; Forstater, J.; Robertson, J. W. F.; Reiner, J. E.; Zhang, H.; Chen, S.; Kasianowicz, J. J. Determining the Physical Properties of Molecules with Nanometer-Scale Pores. ACS Sensors 2018, 3 (2), 251-263.

(29) Howorka, S. Building Membrane Nanopores. Nat. Nanotechnol. 2017, 12 (7), 619-630.

(30) Song, L.; Hobaugh, M. R.; Shustak, C.; Cheley, S.; Bayley, H.; Gouaux, J. E. Structure of Staphylococcal $\alpha$-Hemolysin, a Heptameric Transmembrane Pore. Science 1996, 274 (5294), 1859-1866.

(31) Menestrina, G. Ionic Channels Formed by Staphylococcus aureus Alpha-Toxin: VoltageDependent Inhibition by Divalent and Trivalent Cations. J Membr Biol. 1986, 90(2), 177190.

(32) Reiner, J. E.; Kasianowicz, J. J.; Nablo, B. J.; Robertson, J. W. F. Theory for Polymer Analysis Using Nanopore-Based Single-Molecule Mass Spectrometry. Proc. Natl. Acad. Sci. U. S. A. 2010, 107 (27), 12080-12085.

(33) Krasilnikov, O. V.; Bezrukov, S. M. Polymer Partitioning from Nonideal Solutions into Protein Voids. Macromolecules 2004, 37 (7), 2650-2657.

(34) Jackson, J. D. Classical Electrodynamics, 3rd ed.; John Wiley \& Sons, 1998.

(35) Getfert, S.; Töws, T.; Reimann, P. Reluctance of a Neutral Nanoparticle to Enter a Charged Pore. Phys. Rev. E - Stat. Nonlinear, Soft Matter Phys. 2013, 88 (5), 052710.

(36) Dolinsky, T. J.; Czodrowski, P.; Li, H.; Nielsen, J. E.; Jensen, J. H.; Klebe, G.; Baker, N. A. PDB2PQR: Expanding and Upgrading Automated Preparation of Biomolecular Structures for Molecular Simulations. Nucleic Acids Res. 2007, 35 (SUPPL.2), W522-W525.

(37) Aguilella-Arzo, M.; Queralt-Martín, M.; Lopez, M. L.; Alcaraz, A. Fluctuation-Driven Transport in Biological Nanopores. A 3D Poisson-Nernst-Planck Study. Entropy 2017, 19 (3), 116.

(38) Aguilella-Arzo, M.; Aguilella, V. M. Access Resistance in Protein Nanopores. A StructureBased Computational Approach. Bioelectrochemistry 2020, 131, 107371.

(39) Cai, Q.; Ye, X.; Luo, R. Dielectric Pressure in Continuum Electrostatic Solvation of Biomolecules. Phys. Chem. Chem. Phys. 2012, 14 (45), 15917-15925.

(40) Guyer, J. E.; Wheeler, D.; Warren, J. A. FiPy: Partial Differential Equations with Python. Comput. Sci. Eng. 2009, 11 (3), 6-15. 
(41) Rossum, G. Van; Drake, F. L. Python Reference Manual http://www.python.org/doc/ref/ (accessed Sep 1, 2020).

(42) Geuzaine, C.; Remacle, J. F. Gmsh: A 3-D Finite Element Mesh Generator with Built-in Pre- and Post-Processing Facilities. Int. J. Numer. Methods Eng. 2009, 79 (11), 13091331.

(43) Geus, R.; Arbenz, P. PySparse and PyFemax : A Python Framework for Large Scale Sparse Linear Algebra. Computing 2003, 1-10.

(44) Aksimentiev, A.; Schulten, K. Imaging alpha-hemolysin with molecular dynamics: ionic conductance, osmotic permeability, and the electrostatic potential map., Biophys. J. 2005, $88(6), 3745-61$.

(45) Aguilella-Arzo, M.; Aguilella, V. M. Continuum Electrostatic Calculations of the pKa of Ionizable Residues in an Ion Channel: Dynamic vs. Static Input Structure. Eur. Phys. J. E 2010, 31 (4), 429-439.

(46) Misakian, M.; Kasianowicz, J. J. Electrostatic Influence on Ion Transport through the AHL Channel. J. Membr. Biol. 2003, 195 (3), 137-146.

(47) Krasilnikov, O. V.; Capistrano, M. F. P.; Yuldasheva, L. N.; Nogueira, R. A. Influence of Cys130 S. Aureus Alpha-Toxin on Planar Lipid Bilayer and Erythrocyte Membranes. J. Membr. Biol. 1997, 156 (2), 157-172.

(48) Arnold, K.; Herrmann, A.; Pratsch, L.; Gawrisch, K. The Dielectric Properties of Aqueous Solutions of Poly(Ethylene Glycol) and Their Influence on Membrane Structure. Biochim. Biophys. Acta 1985, 815 (3), 515-518.

(49) Sarode, A. V.; Kumbharkhane, A. C. Dielectric Relaxation Study of Poly(Ethylene Glycols) Using TDR Technique. J. Mol. Liq. 2011, 164 (3), 226-232.

(50) Lee, H.; Venable, R. M.; MacKerell, A. D.; Pastor, R. W. Molecular Dynamics Studies of Polyethylene Oxide and Polyethylene Glycol: Hydrodynamic Radius and Shape Anisotropy. Biophys. J. 2008, 95 (4), 1590-1599.

(51) Gu, L.Q.; Cheley, S.; Bayley, H. Electroosmotic enhancement of the binding of a neutral molecule to a transmembrane pore. Proc Natl Acad Sci U S A. 2003, 100 (26), 1549815503.

(52) Piguet, F.; Discala, F.; Breton, M.F.; Pelta, J.; Bacri, L.; Oukhaled, A. Electroosmosis through $\alpha$-Hemolysin That Depends on Alkali Cation Type. J Phys Chem Lett. 2014, 5 (24), 4362-4367. 